\title{
Redox regulation of cellular stress response in multiple sclerosis
}

\author{
G. Pennisi ${ }^{a}$, C. $_{\text {Cornelius }}{ }^{\mathrm{b}}$, M.M. Cavallaro ${ }^{\mathrm{b}}$, A. Trovato Salinaro ${ }^{\mathrm{b}}$, M.T. Cambria $^{\mathrm{b}}$, M. Pennisi ${ }^{\mathrm{b}}$, R. Bella $^{\mathrm{a}}$, \\ P. Milone ${ }^{\mathrm{c}}$, B. Ventimiglia $^{\mathrm{d}}$, M.R. Migliore ${ }^{\mathrm{b}}$, L. Di Renzo $^{\mathrm{e}}$, A. De Lorenzo $^{\mathrm{e}}$, V. Calabrese $^{\mathrm{b}, *}$ \\ a Department of Neuroscience, University of Catania, I-95125 Catania, Italy \\ ${ }^{\mathrm{b}}$ Department of Chemistry, University of Catania, I-95125 Catania, Italy \\ ' Department of Radiological Sciences, University of Catania, I-95125 Catania, Italy \\ ${ }^{\mathrm{d}}$ Department of Medicine and Surgery, University of Catania, I-95125 Catania, Italy \\ ${ }^{\mathrm{e}}$ Division of Human Nutrition, Department of Neuroscience, University of Rome Tor Vergata, I-00133 Rome, Italy
}

\section{A R T I C L E I N F O}

\section{Article history:}

Received 6 May 2011

Accepted 22 July 2011

Available online 30 July 2011

This paper is dedicated to the memory of Claudio Cavazza, who died on June 6th, while this manuscript was in preparation.

\section{Keywords:}

Multiple sclerosis

Oxidative stress

Vitagenes

Heat shock proteins

Sirtuins

\begin{abstract}
A B S T R A C T
Multiple sclerosis (MS) is an autoimmune-mediated neurodegenerative disease with characteristic foci of inflammatory demyelination in the brain, spinal cord, and optic nerves. Recent studies have demonstrated not only that axonal damage and neuronal loss are significant pathologic components of MS, but that this neuronal damage is thought to cause the permanent neurologic disability often seen in MS patients. Emerging finding suggests that altered redox homeostasis and increased oxidative stress, primarily implicated in the pathogenesis of MS, are a trigger for activation of a brain stress response. Relevant to maintenance of redox homeostasis, integrated mechanisms controlled by vitagenes operate in brain in preserving neuronal survival during stressful conditions. Vitagenes encode for heat shock proteins (Hsp) Hsp32, Hsp70, the thioredoxin and the sirtuin protein systems. In the present study we assess stress response mechanisms in the CSF, plasma and lymphocytes of control patients compared to MS patients. We found that the levels of vitagenes Hsp72, Hsc70, HO-1, as well as oxidative stress markers carbonyls and hydroxynonenals were significantly higher in the blood and CSF of MS patients than in control patients. In addition, an increased expression of Trx and sirtuin 1, together with a decrease in the expression of TrxR were observed. Our data strongly support a pivotal role for redox homeostasis disruption in the pathogenesis of MS and, consistently with the notion that new therapies that prevent neurodegeneration through nonimmunomodulatory mechanisms can have a tremendous potential to work synergistically with current MS therapies, unravel important targets for new cytoprotective strategies.
\end{abstract}

(c) 2011 Elsevier Inc. All rights reserved.

\section{Introduction}

Multiple sclerosis (MS) is an autoimmune-mediated neurodegenerative disease with characteristic foci of inflammatory demyelination in the brain, spinal cord, and optic nerves. Recent studies have demonstrated not only that axonal damage and neuronal loss are significant pathologic components of MS and experimental autoimmune encephalomyelitis (EAE), but that this neuronal damage is thought to cause the permanent neurologic disability often seen in MS patients. Current treatments for MS involve immunomodulation, which can reduce the incidence of inflammatory relapses. However, existing therapies are often not fully effective, and limited evidence suggests that these therapies prevent the long-term neuronal damage and physical disability of MS patients $[1,2]$. New therapies that prevent neurodegeneration

\footnotetext{
* Corresponding author at: Department of Chemistry, Faculty of Medicine, University of Catania, Italy. Tel.: +39 095738 4067; fax: +39 095580138.

E-mail address: calabres@unict.it (V. Calabrese).
}

through nonimmunomodulatory mechanisms have a tremendous potential to work synergistically with current MS therapies [3]. MS pathology is characterized by perivenous infiltration of lymphocytes and macrophage leading to damage of myelin and axons in the brain and spinal cord, which underlie the clinical disease course usually occurring with recurrent and reversible episodes of neurological dysfunction affecting one or several sites, during late adolescence and early adulthood; this form is called relapsingremitting form (RR) and it is the most prevalent. Usually, approximately 20 years later, this clinical pattern transforms into a secondary progressive phase with continuous and progressive neurological decline [4].

Although evidence indicates that MS is a multifactorial disease caused by a complex interplay between genetic and environmental factors, it is still unclear which are the causes or the factors that contribute to its unpredictable course. It is generally accepted that, virus infections of the CNS, vascular factors and/or disturbed immune mechanisms are implicated in the pathogenesis of MS [5,6]. Once triggered, the immune system attacks and destroys myelin and the myelin-forming cell, leading to the pathological hallmarks of 
MS: the classical actively demyelinating lesions, the cortical demyelination and the diffuse white matter injury, the latter particularly evident in the later stage of the disease [6,7]. Existing evidence indicates that CNS responds to the attack by immune cells and their secreted products through modulation of its metabolism and gene expression [8]. In addition, cytokines, immunoglobulins, and complement complexes may elicit a survival response in the oligodendrocytes, involving the induction of endogenous heat shock proteins and other protective molecules, which indicates that redox systems and therefore the oxidant/antioxidant balance in these cells are of great importance in MS [9-12].

The adaptation and survival of cells and organisms requires the ability to sense proteotoxic insults and to coordinate protective cellular stress response pathways and chaperone networks related to protein quality control [13]. Despite the abundance and apparent capacity of chaperones and other components of homeostasis to restore folding equilibrium, brain cells appears poorly adapted for chronic proteotoxic stress which increases in neurodegenerative diseases such as MS [14]. In these conditions, a decline in biosynthetic and repair activities that compromises the integrity of the proteome is strongly influenced by protective genes called "vitagenes" that control aging, thus linking stress and protein homeostasis with the health antidegenerative mechanisms [15]. Vitagenes encode for heat shock proteins (Hsp) Hsp32, Hsp70, the thioredoxin and the sirtuin protein systems. In the CNS, Hsps synthesis occurs after hyperthermia, alterations in the intracellular redox environment, exposure to heavy metals, amino acid analogs or cytotoxic drugs [15]. While prolonged exposure to conditions of extreme stress is detrimental and can induce cell death, Hsps synthesis can result in stress tolerance and cytoprotection in various metabolic diseases and injuries, including hypoxia, stroke, epilepsy, cell and tissue trauma, neurodegenerative disease and aging [14]. Sirtuins (SIRTs) represent a highly conserved gene family encoding NAD+-dependent deacetylases, originally found to deacetylate histones leading to increased DNA stability and prolonged survival in yeast and higher organisms, including mammals. Members of the sirtuin family have since been found to function as deacetylases for numerous protein targets involved in many cellular pathways, including cellular stress responses, apoptosis, and axonal degeneration. The activity of sirtuins can be regulated by other compounds that can serve as activators (e.g., plant polyphenols) that increase enzyme activity by lowering the $\mathrm{Km}$ of its substrates or that can serve as inhibitors (e.g., sirtinol) $[15,16]$. Neuroprotection mediated by SIRT1 activation has been demonstrated in axotomized dorsal root ganglion neurons and in neurons containing the Huntingtin gene mutation responsible for neurodegeneration in Huntington disease. Thus, pharmacological modulation of cellular stress pathways has emerging implications for the treatment of human diseases, including cardiovascular disease, cancer and neurodegenerative disorders [17,18].

The present study was undertaken in order to investigate systemic stress response and the associated oxidative stress measured through the determination of markers of protein and lipid oxidation in plasma, lymphocytes and CSF of patients with active MS, as compared to age-matched controls, in order to gain a better insight into the molecular mechanisms regulating the cellular stress response during the progression of the disease and, as such, provide a potential target for novel cytoprotective strategies impacting the clinical settings of this degenerative disease.

\section{Materials and methods}

\subsection{Ethical permission}

The study was conducted according to guidelines of local Ethics Committee, and informed consent was obtained from all patients.

\subsection{Patients}

\subsubsection{Multiple sclerosis patients}

Twenty-six patients with an age range of 20-60 years were used as the "test" group for this study. The mean disease duration was $3.9 \pm 2.0$ years. All these patients had a confirmed clinical diagnosis of MS according to the diagnostic criteria of McDonald et al. [19]. Furthermore, all the subjects were clinically classified having the relapsing remitting form of MS according to the criteria described in Lublin and Reingold [20]. The patients had not been undergoing corticosteroid or immunosuppressive treatment for at least 2 months before the CSF samples were collected.

\subsubsection{Control patients}

Twenty control patients, with an age range of 30-60 years, underwent lumbar puncture because of suspected subarachnoid hemorrage, pseudotumor cerebri, oculomotor palsies, or other indications in the usual neurological survey. Laboratory and neuroimaging tests were normal. Therefore the final diagnosis was mainly tension headache or conversion disorder.

\subsection{Sampling}

Blood was collected from controls and patients by venipuncture from an antecubital vein into tubes containing $500 \mathrm{mM}$ EDTA as an anticoagulant. Immediately after sampling, $1 \mathrm{ml}$ the blood was centrifuged at $10,000 \times \mathrm{g}$ for $10 \mathrm{~min}$ at $4{ }^{\circ} \mathrm{C}$ to separate serum from red blood cells and $4 \mathrm{~mL}$ were utilized for lymphocytes purification. Lymphocytes from peripheral blood were purified using the Ficoll Paque System following the procedure provided by the manufacturer (GE Healthcare, Piscataway, NJ, USA). CSF was obtained (on ice) from all subjects by lumbar spinal tap. The CSF samples were immediately centrifuged at $10,000 \times g$ for $3 \mathrm{~min}$ at $4{ }^{\circ} \mathrm{C}$ to remove any contaminating cells and kept on ice until the biochemical assays were performed.

Chemical analysis of CSF showed no heme present in the CSF in any of the control or MS CSF samples. CSF lymphocytes count was $1.05 \pm 1.17$ in the first MS CSF sample and $1.04 \pm 1.15$ in second ones. The control group lymphocytes count was $1 \pm 0.12$.

\subsection{Western blot analysis}

Carbonyls (DPNH), HNE, Hsp60, Hsc70, Hsp-72, HO-1, HO-2, Trx, TrxR-1, Sirt-1 were evaluated by Western blot analyses. In brief, proteins $(40 \mu \mathrm{g})$ for each sample were separated by a $12.5 \%$ SDS-PAGE and electroblotted ( $1 \mathrm{~h}$ at $100 \mathrm{~V}$ ) to nitrocellulose membranes (Bio-Rad) using $25 \mathrm{mM}$ Tris, $192 \mathrm{mM}$ glycine and 20\% $(\mathrm{v} / \mathrm{v})$ methanol. Equal protein loading was confirmed by staining with $0.2 \%(\mathrm{v} / \mathrm{v})$ Ponceau $\mathrm{S}$ in $7 \%$ acetic acid. Blotted membranes were blocked with $3 \%$ non fat dry milk in phosphate-buffered saline and challenged with appropriate primary antibodies, namely anti-Hsc70, monoclonal antibody (Santa Cruz Biotech. Inc., Santa Cruz, CA, USA), that recognizes the constitutive form of Hsp 70, or with a monoclonal anti-Hsp72 antibody (RPN 1197, Amersham) that recognizes only the inducible form, anti-DPNH (V0401 DAKO, Glostrup, Denmark), anti-HNE (HNE11-S Alpha Diagnostic International, San Antonio, TX, USA), anti-HO-1, antiHO-2 (Stressgen, Victoria, BC, Canada) anti-Trx, anti TrxR1 and anti Sirt-1 rabbit polyclonal antibody (Santa Cruz Biotech. Inc., Santa Cruz, CA, USA), for $1 \mathrm{~h}$ at room temperature. Immunodetection of Hsp60 was performed using a polyclonal goat antibody sc-1052 (Santa Cruz Biotech. Inc., Santa Cruz, CA, USA). Unbound antibodies were removed by washing TBS-T for $5 \mathrm{~min}$. The membranes were then incubated with horseradish peroxidase-conjugated secondary antibody diluted $1: 10,000$. Protein bands were visualized with ECL Plus $^{\mathrm{TM}}$ (Amersham, NJ, USA) according to the manufacturer's 
Table 1

Clinical and demographic data of MS patients and control subjects.

\begin{tabular}{|c|c|c|c|c|c|c|}
\hline & Number of subjects & Age $($ mean $\pm S D)$ & Gender (F/M) & Age at disease onset (mean \pm SD) & Disease duration (mean $\pm \mathrm{SD}$ ) & EDSS $($ mean \pm SD) \\
\hline Patients & 26 & $35.3 \pm 9.9$ & $16 / 10$ & $31.4 \pm 9.2$ & $3.92 \pm 2.08$ & $1.5 \pm 1.18$ \\
\hline Controls & 20 & $36.9 \pm 10.9$ & $12 / 8$ & & & \\
\hline
\end{tabular}

EDSS: Expanded Disability Status Scale.

protocol. Immunoreactive bands were quantified by scanning Western blot-imaged films with a laser densitometer (LKBUltrascan, XL model, Pharmacia, American Instruments, Haverhill, MA, USA). Molecular weights of the proteins detected were determined using a standard curve obtained with proteins of know molecular weight.

\subsection{Protein measurements}

Samples protein concentrations were determined by the bicinchoninic acid method [21].

\subsection{Statistical analysis}

All results are expressed as means \pm standard error of mean. Each experiment was performed, unless otherwise specified, in triplicate. Data were analyzed by one-way analysis of variance, followed by inspection of all differences by Duncan's new multiplerange test. Differences were considered significant at $P<0.05$.

\section{Results}

Multiple sclerosis (MS) is a chronic inflammatory demyelinating disease of the central nervous system (CNS) of unclear etiology, characterized by perivenous infiltration of lymphocytes and macrophage leading damage of myelin and axons in the brain and spinal cord [1]. Clinical disease usually occurs with recurrent and reversible episodes of neurological dysfunction affecting one or several sites, during late adolescence and early adulthood; this form being the most prevalent is called relapsing-remitting form (RR) and was chosen in this study. Table 1 summarizes clinical data of patients and control subjects. As shown, the mean age at disease onset was $31.4 \pm 9.2$ years with a disease duration of $3.92 \pm 2.08$ years, a relatively short duration typical of the relapsing remitting form not yet changed into the secondary progressive form. The mean value of Expanded Disability Status Scale (EDSS), which the most used method of quantifying disability in multiple sclerosis, was $1.5 \pm 1.18$ and this indicates that patients have a low degree of disability linked to the short period of disease. Brain MRI from MS patients presenting two hypointense areas distributed bilaterally on the white matter of the semioval center $(A)$, enhanced by gadolinium injection $(B)$ is shown in Fig. $1 \mathrm{~A}$ and $\mathrm{B}$.

The heat shock response contributes to establishing a cytoprotective state in a wide variety of human diseases, including inflammation, cancer, aging and neurodegenerative disorders. The vitagene family is composed of the heat shock proteins HO-1 (also called Hsp32), Hsp70, by the thioredoxin system and by sirtuin proteins [22-24]. We therefore evaluated the expression levels of cellular stress response proteins in the CSF, plasma and lymphocytes in control and in MS patients. Western blot analysis of CSF probed for heme oxygenase- 1 is reported in Fig. 2. HO- 1 expression is significantly $(P<0.05)$ increased in the CSF of MS patients, compared to controls. This finding was associated with increased levels of protein oxidation (Fig. 3A) as well as lipid oxidation (Fig. 3C). Protein oxidation was evaluated by measuring the amount of protein carbonyls (DNPH). Protein carbonyls most often are detected by two methods, i.e., derivatization with 2,4-dinitrophenylhydrazine
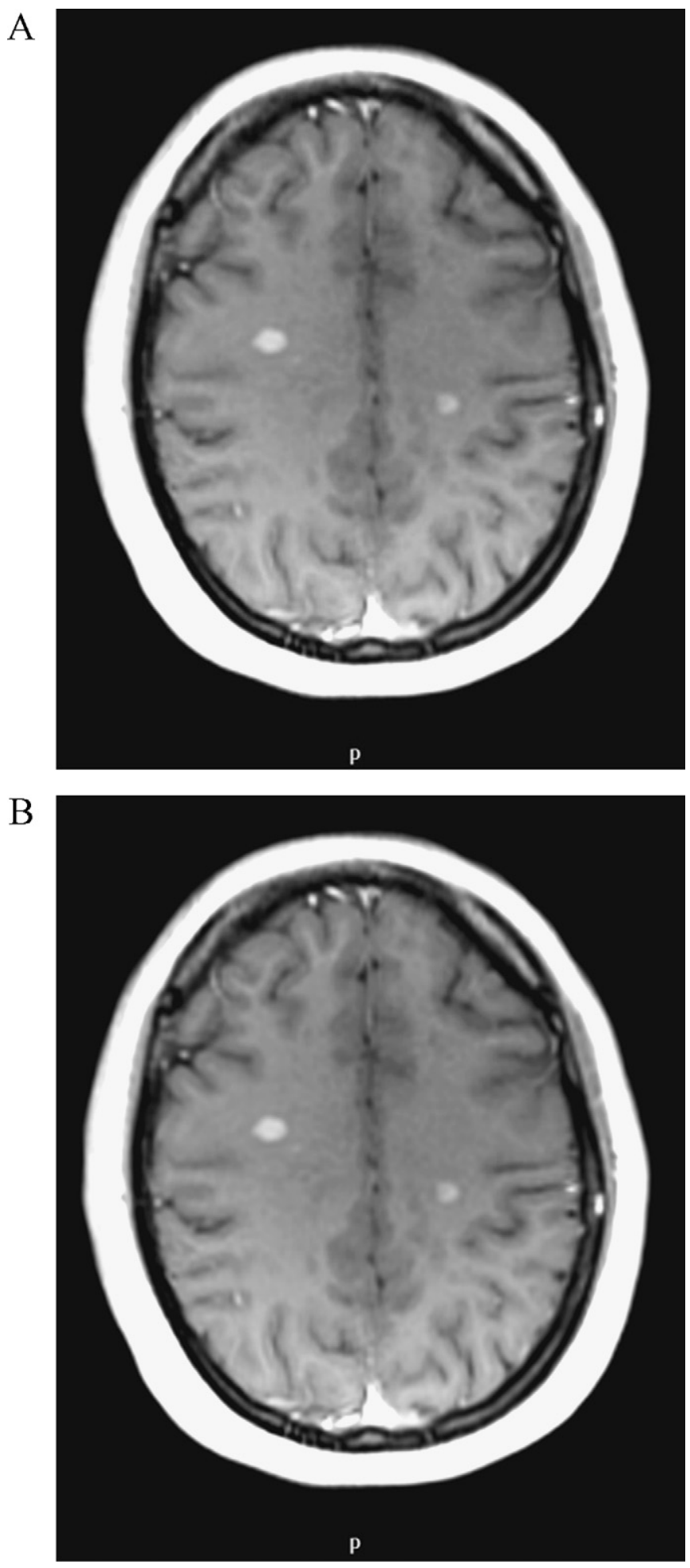

Fig. 1. Brain MRI from MS patients: axial T1WI shows two hypointense areas on the white matter of the semioval center bilaterally $(A)$ that present enhancement after gadolinium injection (B). 

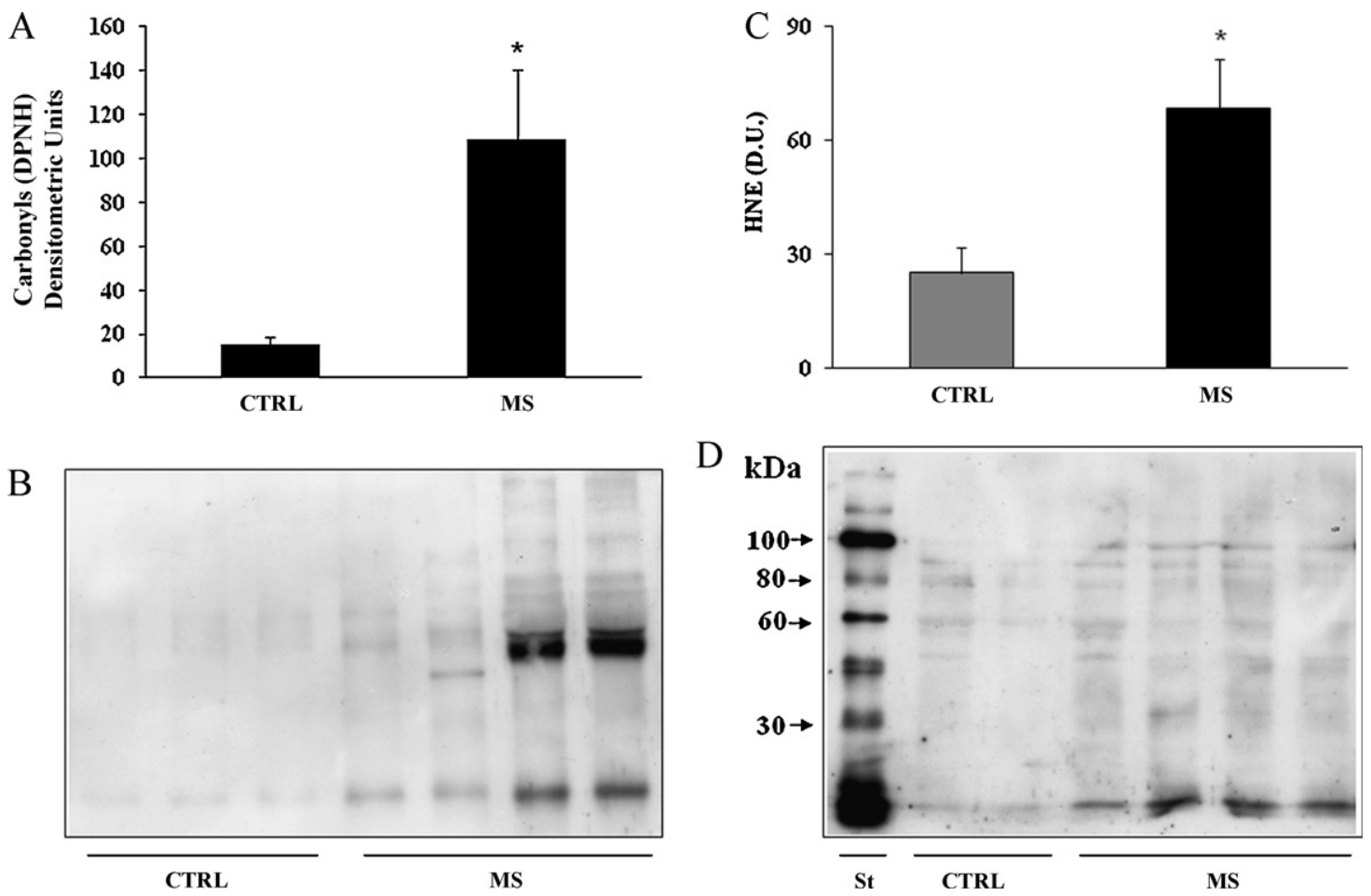

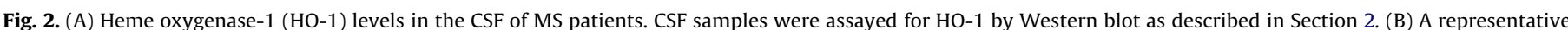

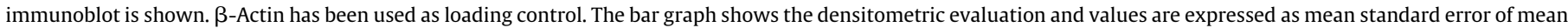

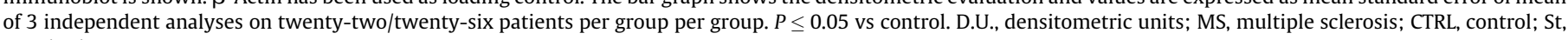
standard.

(DNPH), followed by immunochemical detection with an antibody against the resulting protein hydrazone, or formation of the Schiff base by biotin hydrazide followed by detection of the protein-bound imine with enzyme- or fluorophore-linked avidin or streptavidin. Here we describe the DNPH-based detection method that is routinely used for detection of carbonylated proteins. In the DNPH method, samples are derivatized with DNPH. DNPH reacts with carbonyl groups to form protein-resident 2,4-dinitrophenylhydrazone (DNP), which is then detected using commercially available anti-DNP antibodies (Fig. 3B). As shown, protein carbonyls were found at a significantly $(P<0.05)$ higher level in the CSF of MS patients than in

A

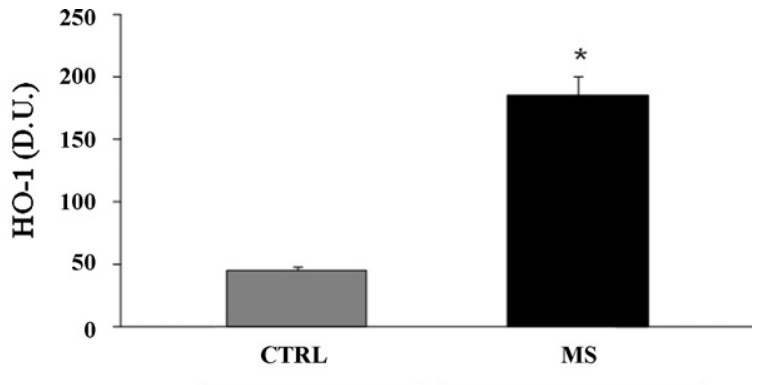

B

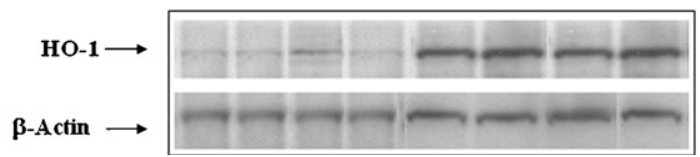

Fig. 3. Protein carbonyls and HNE (4-hydroxy-2-nonenals) levels in the CSF of MS patients. CSF samples were assayed for protein carbonyls (A, B), and HNE (C, D) by Western blot as described in Section 2. Representative immunoblots are shown (B, D). The bar graph shows the densitometric evaluation and values are expressed as mean standard error of mean of 3 independent analyses on twenty-two/twenty-six patients per group. $P \leq 0.05$ vs control. D.U., densitometric units; MS, multiple sclerosis; CTRL, control. control subjects. Furthermore, one measure of oxidative marker is lipid oxidation, indexed by HNE [25,26], which can also occur in brain under oxidative stress [27]. HNE, formed from arachidonic acid or other unsaturated fatty acids following free radical attack, binds by Michael addition to proteins, particularly at cysteine, histidine, or lysine residues [27]. Examination of HNE levels in the CSF of MS patients showed a significant $(P<0.05)$ elevation of protein-bound HNE as compared to control group (Fig. 3D).

The $70 \mathrm{kDa}$ family of stress proteins is one of the most extensively studied cytoprotective system. Included in this family are the constitutive isoform, Hsc70 (heat shock cognate), and the inducible isoform, Hsp70 (also referred to as Hsp72). Figs. 4 and 5 show a significant $(P<0.05)$ increase in the expression level of

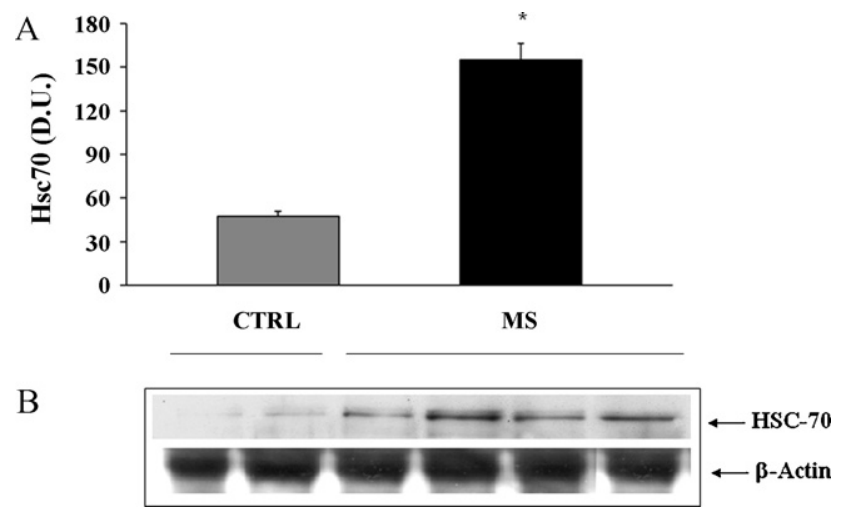

Fig. 4. (A) Heat shock protein 70 (Hsc70) levels in MS lymphocytes. Lymphocytes were assayed for Hsc-70 by Western blot as described in Section 2. (B) A representative immunoblot is shown. $\beta$-Actin has been used as loading control. The bar graph shows the densitometric evaluation and values are expressed as mean standard error of mean of 3 independent analyses on twenty-two/twenty-six patients per group. $P \leq 0.05$ vs control. D.U., densitometric units; MS, multiple sclerosis; CTRL, control. 


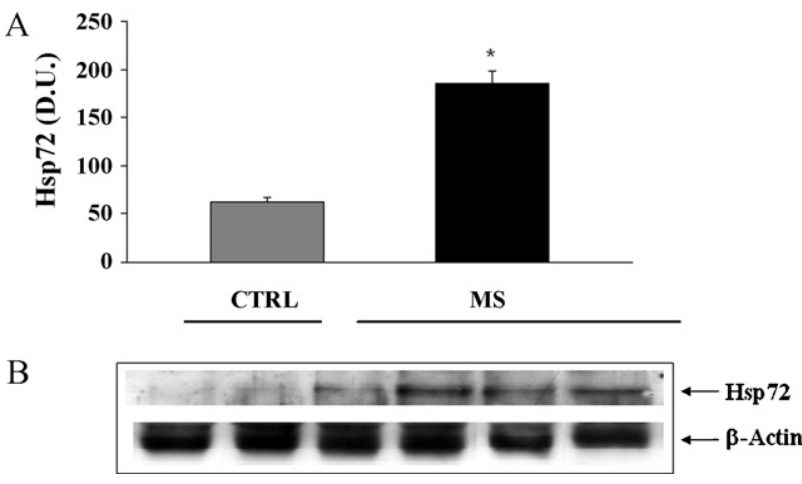

Fig. 5. (A) Heat shock protein 72 (Hsp72) levels in multiple sclerosis patients. Lymphocytes samples were assayed for Hsc-72 by Western blot as described in Section 2. (B) A representative immunoblot is shown. $\beta$-Actin has been used as loading control. The bar graph shows the densitometric evaluation and values are expressed as mean standard error of mean of 3 independent analyses on twentytwo/twenty-six patients per group. $P \leq 0.05$ vs control. D.U., densitometric units; MS, multiple sclerosis; CTRL, control.

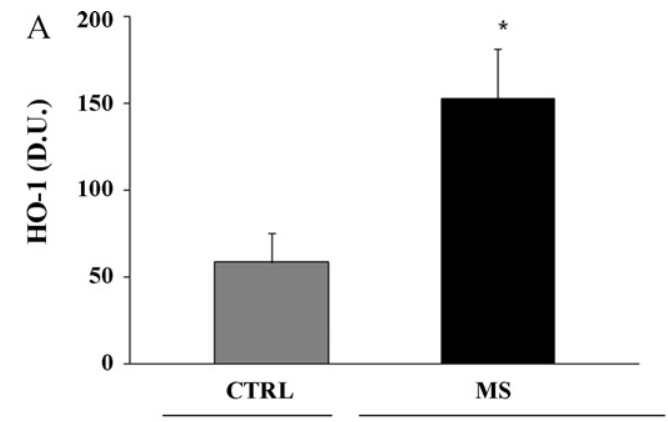

B

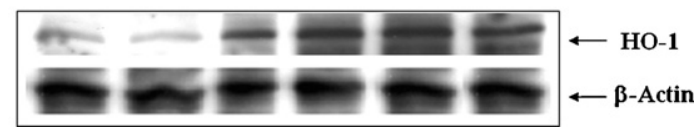

Fig. 6. (A) Heme oxygenase-1 (HO-1) levels in lymphocytes of MS patients. Lymphocytes samples were assayed for HO-1 by Western blot as described in Section 2. (B) A representative immunoblot is shown. $\beta$-Actin has been used as loading control. The bar graph shows the densitometric evaluation and values are expressed as mean standard error of mean of 3 independent analyses on twentytwo/twenty-six patients per group. $P \leq 0.05$ vs control. D.U., densitometric units; MS, multiple sclerosis; CTRL, control.

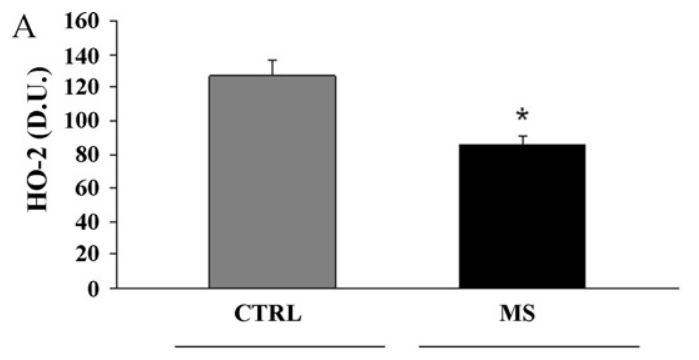

B

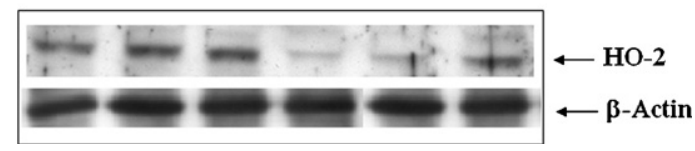

Fig. 7. (A) Heme oxygenase-2 (HO-2) levels in lymphocytes of MS patients. Lymphocytes samples were assayed for $\mathrm{HO}-2$ by Western blot as described in Section 2. (B) A representative immunoblot is shown. $\beta$-Actin has been used as loading control. The bar graph shows the densitometric evaluation and values are expressed as mean standard error of mean of 3 independent analyses on twentytwo/twenty-six patients per group. $P \leq 0.05$ vs control. D.U., densitometric units; MS, multiple sclerosis; CTRL, control.
Hsc70 and Hsp70, respectively, in lymphocytes of MS patients with respect to controls (Figs. $4 \mathrm{~A}$ and $5 \mathrm{~A}$ ). Representative immunoblots are also shown (Figs. $4 \mathrm{~B}$ and $5 \mathrm{~B}$ ).

Heme oxygenase-1 (HO-1), also referred to as Hsp32, belongs to the Hsp family and protects brain cells from oxidative stress by degrading toxic heme into free iron, carbon monoxide and biliverdin [17,24]. This latter is then reduced by biliverdin reductase (BVR) into bilirubin (BR), a linear tetrapyrrole with antioxidant properties; very recently, BR has been shown to effectively counteract also nitrosative stress, due to its ability to bind and inactivate NO and RNS [14,17]. The constitutive isoform of heme oxygenase is HO-2 [28,29]. We evaluated the expression of both isoforms in lymphocytes of MS patients and in controls. As shown in Fig. 6, HO-1 protein expression was higher in MS samples compared to control. Notably, increase in HO-1 was associated with a significant $(P<0.05)$ decrease in $\mathrm{HO}-2$ expression in the same experimental group (Fig. 7). Analysis of lymphocytes in MS patients, compared to control group, revealed also a significant $(P<0.05)$ increase in thioredoxin expression, whereas expression of the related enzyme thioredoxin reductase- 1 was decreased (Figs. 8A, B and 9A, B). These changes were associated with significantly $(P<0.05)$ increased formation of protein carbonyls (Fig. 10A and B) and HNE (Fig. 10C and D). Consistently to the observed changes in MS lymphocytes, analysis of plasma in MS patients showed higher and significant $(P<0.05)$ expression levels of HO-1, Hsp60 than in control subjects (Figs. 11 and 12), while no difference was found to a significant extent between the two experimental groups in Hsp70 protein levels (Fig. 13). Interestingly, we found in the plasma significantly $(P<0.05)$ higher levels of sirtuin-1 protein in MS patients than in control group (Fig. 14). As far as we are concerned this is the first evidence of changes in SIRT1 expression in MS, although at the moment we can not exclude that this might not be a specific alteration of this progressive inflammatory neurodegenerative disease. A summary of changes of all stress response proteins investigated in CSF, lymphocytes and plasma are reported in Table 2.

\section{Discussion}

Multiple sclerosis is a relatively common and etiologically unknown disease with no cure. It is the leading cause of neurological disability in young adults, affecting over two million people worldwide. Traditionally, MS has been considered a chronic, inflammatory disorder of the central white matter in which ensuing demyelination results in physical disability [30].

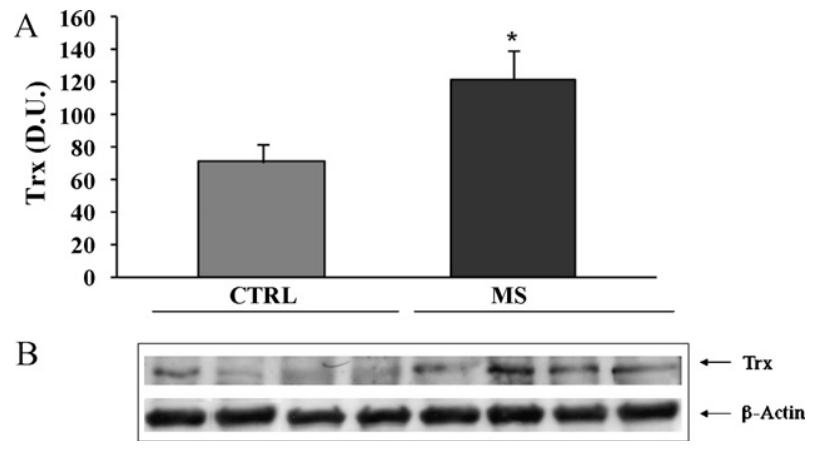

Fig. 8. (A) Thioredoxin (Trx) levels in lymphocytes from multiple sclerosis patients. Lymphocytes samples were assayed for Trx by Western blot as described in Section 2. (B) A representative immunoblot is shown. $\beta$-Actin has been used as loading control. The bar graph shows the densitometric evaluation and values are expressed as mean standard error of mean of 3 independent analyses on twenty-two/twentysix patients per group. $P \leq 0.05$ vs control. D.U., densitometric units; MS, multiple sclerosis; CTRL, control. 


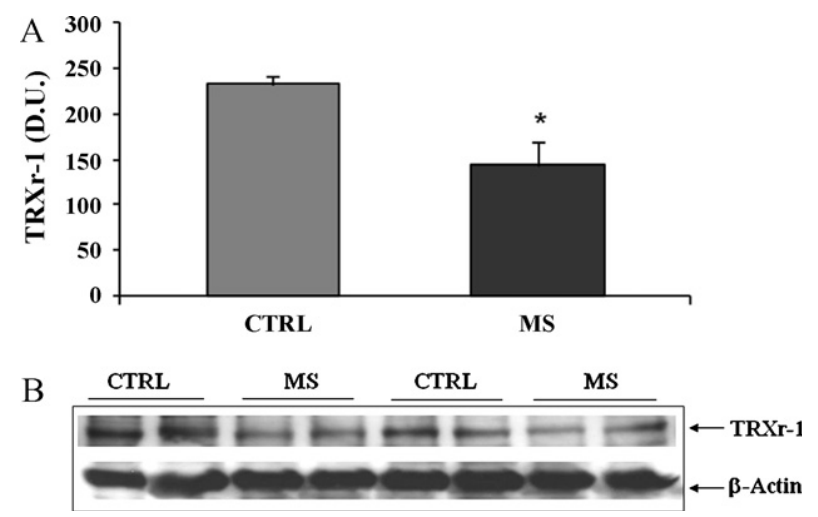

Fig. 9. (A) Thioredoxin reductase-1 (TrxR-1) levels in lymphocytes from patients with multiple sclerosis. Lymphocytes samples were assayed for TrxR-1 by Western blot as described in Section 2. (B) A representative immunoblot is shown. $\beta$-Actin has been used as loading control. The bar graph shows the densitometric evaluation and values are expressed as mean standard error of mean of 3 independent analyses on twenty-two/twenty-six patients per group. $P \leq 0.05$ vs control. D.U., densitometric units; MS, multiple sclerosis; CTRL, control.

Recently, MS has become increasingly viewed not only an inflammatory disease, but also a neurodegenerative disorder in which axonal injury, neuronal loss and atrophy of the central nervous system lead to permanent neurological and clinical disability [31]. There are three main types of MS, defined as: relapsing-remitting (RR), secondary progressive (SP) and primaryprogressive (PP) with progressive-relapsing (PR) recently distinguished as an additional subtype [31,32]. There is evidence that MS is characterized not only by immune mediated inflammatory reactions but also by neurodegenerative processes [33,34]. A variety of studies, supporting a role for oxidative stress in MS, indicate that endogenous antioxidants are decreased in MS, and damage to mitochondria induced by lipid peroxidation can lead to further ROS generation [35-39]. However, up-regulation of antioxidants in astrocytes and macrophages in active MS lesions
[30] as well as in leukocytes suggests that endogenous protection from oxidative damage could limit the deleterious consequences of the oxidative stress per se [40]. Interestingly, exogenously administrated antioxidants have shown some beneficial effects in animal studies of MS but no clinical studies support the use of such therapy [41-44]. Furthermore, the possible links between MS and redox balance perturbation is indicated by increased malonaldehyde and F2-iP levels in MS patients as compared to control individuals with other neurological disorders [11] and, in addition, an inappropriate expression of Hsps on oligodendrocytes $[36,45]$. This last event could represent a possible initiating factor at the level of MS lesions, capable of modulating the subsequent susceptibility or resistance of cells to oxidative stress. In a previous report we have demonstrated high levels of NO, peroxynitrite, and superoxide in spinal fluid from patients with MS [5,9]. Activated glia secrete RNS products of NO metabolism with superoxide radicals $\left(\mathrm{O}_{2}{ }^{-}\right)$to form peroxynitrite anion $\left(\mathrm{ONOO}^{-}\right)$. RNS can cause nitrosative stress, which results in the destruction of myelin and (myelin-forming) oligodendrocyte cells [46]. Furthermore, the formation of S-nitrosoglutathione (GSNO) can cause GSH depletion, and hence trigger redox dependent changes in cellular signaling as well as modification of key intracellular enzymes, such as chain respiratory complex activities [47].

In this study, significant increases in oxidative stress markers, such as carbonyls and HNE were found in the CSF, peripheral lymphocytes and plasma of MS patients, as compared to control subjects. These changes were associated with induction of stress responsive proteins, such as $\mathrm{HO}-1, \mathrm{Hsp} 72$, Hsp60 and thioredoxin (TRX), measured in CSF, lymphocytes and in plasma. Heme oxygenase is the rate-limiting enzyme in the production of bilirubin. In the past decade the heme oxygenase ( $\mathrm{HO}$ ) system has been focused for its potential significance in maintaining cellular homeostasis. It is located in the endoplasmic reticulum in a complex with NADPH cytochrome $c$ P450 reductase. The reaction catalyzed by $\mathrm{HO}$ is the oxidative cleavage of the heme molecule to form biliverdin and carbon monoxide (CO). Iron is reduced to its ferrous state through the action of NADPH cytochrome $c$ P450
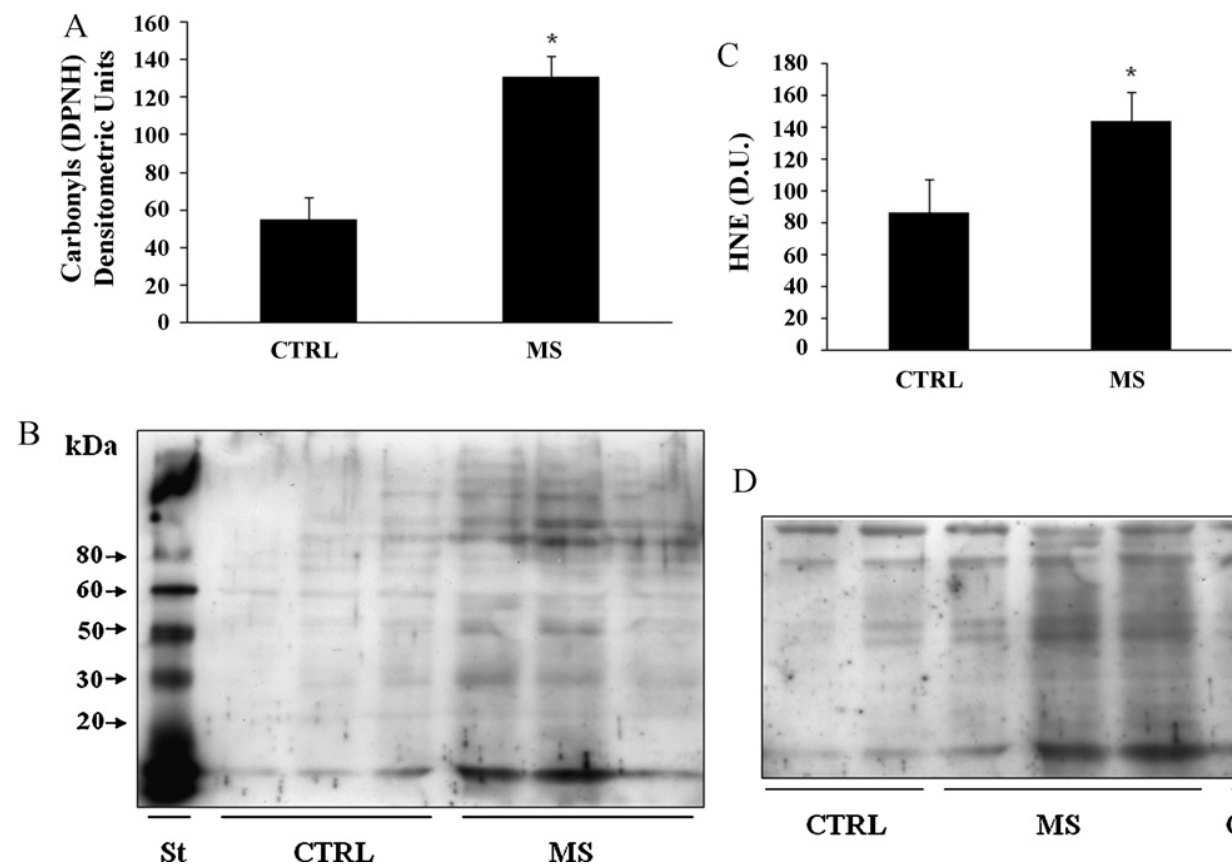

D

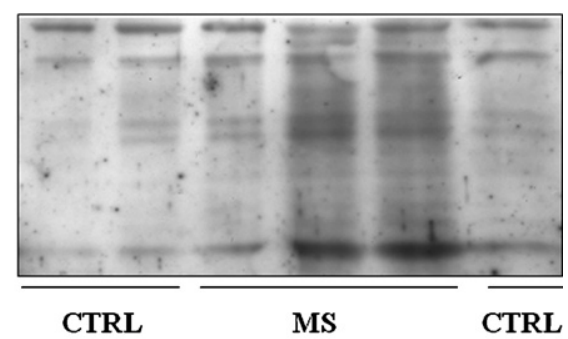

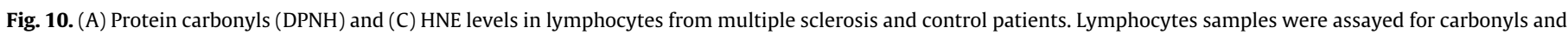

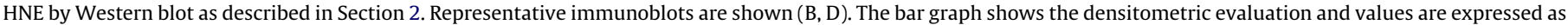

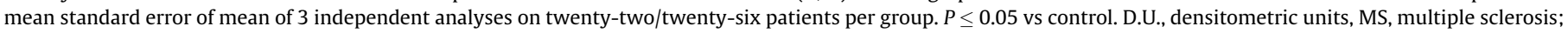
CTRL, control; St, standard. 


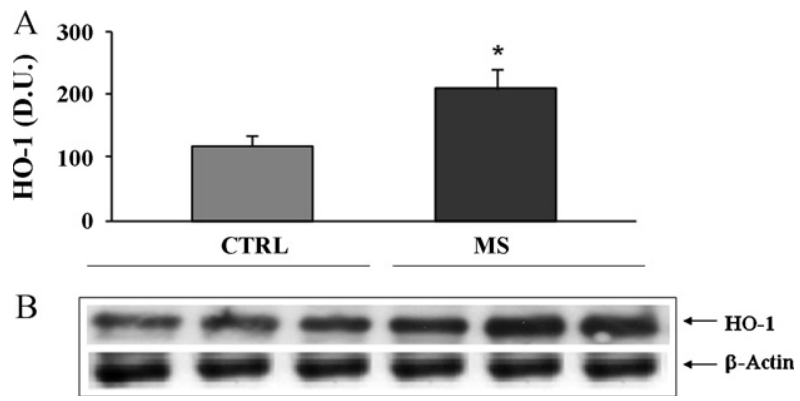

Fig. 11. (A) Heme oxygenase-1 (HO-1) levels in plasma from multiple sclerosis patients. Plasma samples were assayed for HO-1 by Western blot as described in Section 2. (B) A representative immunoblot is shown. $\beta$-Actin has been used as loading control. The bar graph shows the densitometric evaluation and values are expressed as mean standard error of mean of 3 independent analyses on twentytwo/twenty-six patients per group. $P \leq 0.05$ vs control. D.U., densitometric units; MS, multiple sclerosis; CTRL, control.

reductase. Further degradation of biliverdin to bilirubin occurs through the action of a cytosolic enzyme, biliverdin reductase [48]. $\mathrm{HO}$ is present in various tissues with the highest activity in the brain, liver, spleen, and testes. There are three isoforms of heme oxygenase, HO-1 or inducible isoform [13,16,22], HO-2 or constitutive isoform [49], and the recently discovered HO-3, cloned only in rat to date [50]. Elevation of HO-1 expression and activity in MS is probably due to elevated oxidative stress. This finding is consistent with evidence suggesting that the HO- 1 gene is redox regulated and, similar to other antioxidant enzymes [50], this occurs because it contains in its promoter region the antioxidant responsive element (ARE). Therefore, the HO-1 gene undergoes a redox sensitive modulation by transcription factors recognizing specific binding sites within the promoter and distal enhancer regions of the HO- 1 gene $[14,15]$. In addition, heme oxygenase- 1 is rapidly upregulated by oxidative and nitrosative stresses, as well as by glutathione depletion [51].

It has been also suggested that inappropriate stress response within the CNS could influence both the permeability of the bloodbrain barrier and the expression of Hsps, thereby initiating the MS lesion [34,52,53]. In addition, cytokines, immunoglobulins, and complement complexes may elicit a survival response involving the induction of endogenous Hsps and other protective molecules, which indicates that redox dependent mechanisms in these cells are of great importance in MS [54-56]. The heat shock response

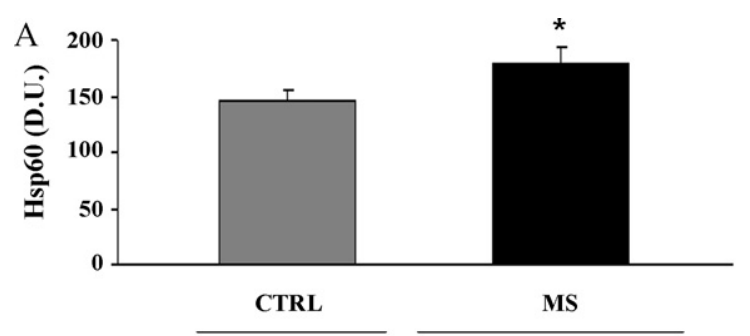

B

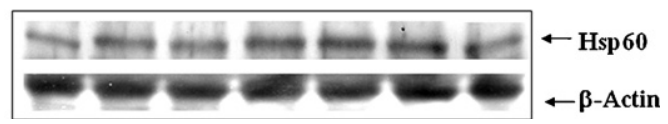

Fig. 12. (A) Heat shock protein 60 (Hsp60) levels in plasma from multiple sclerosis patients. Plasma samples from multiple sclerosis patients and control subjects were assayed for Hsp60 by Western blot as described in Section 2. A representative immunoblot is shown in (B). $\beta$-Actin has been used as loading control. The bar graph shows the densitometric evaluation and values are expressed as mean standard error of mean of 3 independent analyses on twenty-two/twenty-six patients per group. $P \leq 0.05$ vs control. D.U., densitometric units; MS, multiple sclerosis; CTRL, control.
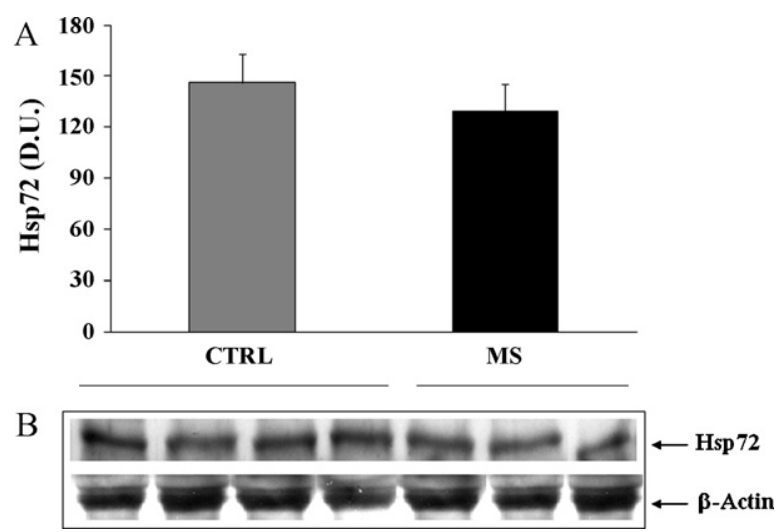

Fig. 13. (A) Heat shock protein 72 (Hsp72) levels in plasma from multiple sclerosis patients. Plasma samples were assayed for Hsp72 by Western blot as described in Section 2. A representative immunoblot is shown in (B). $\beta$-Actin has been used as loading control. The bar graph shows the densitometric evaluation and values are expressed as mean standard error of mean of 3 independent analyses on twentytwo/twenty-six patients per group. $P \leq 0.05$ vs control. D.U., densitometric units; MS, multiple sclerosis; CTRL, control.

contributes to establish a cytoprotective state in a variety of metabolic disturbances and injuries, including stroke, epilepsy, cell and tissue trauma, neurodegenerative disease, and aging $[15,24]$. In mammalian cells, the induction of the heat shock response requires the activation and translocation to the nucleus of one or more heat shock transcription factors (HSFs), which control the expression of a specific set of genes encoding cytoprotective Hsps. Production of Hsps is essential for the folding and repair of damaged proteins, serving thus to promote cell survival [34,52]. Hsps serve as chaperones that bind to other proteins and regulate their conformation, regulate protein movement across membranes or through organelles, or regulate the availability of a receptor or activity of an enzyme. The 70-kDa family of stress proteins is one of the most extensively studied. This family comprises Hsc70 (heatshock cognate, the constitutive form), Hsp70 (the inducible form or Hsp72), and GRP75 (a constitutively expressed glucose-regulated protein found in the endoplasmic reticulum). After a CNS injury, Hsp70 is synthesized at high levels and is present in the cytosol, nucleus, and endoplasmic reticulum [52]. Hsp60 chaperone proteins within mitochondria and, also, protect brain cells against
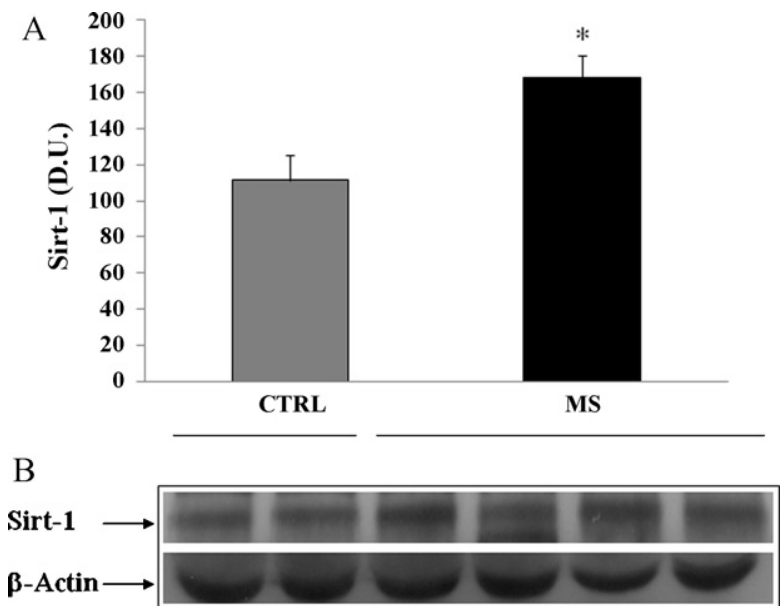

Fig. 14. (A) Sirtuin-1 (Sirt-1) levels in plasma from multiple sclerosis patients Plasma samples were assayed for Sirt-1 by Western blot as described in Section 2. A representative immunoblot is shown in (B). $\beta$-Actin has been used as loading control. The bar graph shows the densitometric evaluation and values are expressed as mean standard error of mean of 3 independent analyses on twenty-two/twentysix patients per group. $P \leq 0.05$ vs control. D.U., densitometric units; MS, multiple sclerosis; CTRL, control. 
Table 2

Summary of different stress response proteins expressed in the CSF, plasma and lymphocytes of MS patients and control subjects.

\begin{tabular}{lllllllll}
\hline MS patients & HO-1 & HO-2 & Hsp60 & Hsc70 & Hsp72 & Trx & TrxR-1 & Sirt-1 \\
\hline Lymphocytes & ++ & -- & & ++ & ++ & ++ & -- & \\
Plasma & ++ & & ++ & & - & & & ++ \\
CSF & ++ & & & & & & & \\
\hline
\end{tabular}

$(++)$ Increased expression; (-) no changes; (- -) decreased expression.

ischemia and seizures in vivo, after viral-induced overexpression [18]. Hsp60 is encoded in the nucleus and resides mainly in the mitochondria [53]. Hsp60 forms the chaperonin complex, which is implicated in protein folding and assembly within the mitochondria under normal conditions [53]. Hsp60 together with its cochaperonin Hsp10 produce a large, efficient protein-editing machinery that facilitates proper folding and assembly of mitochondrial-imported proteins and corrects misfolded polypeptides generated under mitochondrial oxidative stress [57]. Mutations in the HSPD1 gene encoding Hsp60 have recently been found to underlie spastic paraplegia 13 (SPG13 [MIM 605280]), an autosomal-dominant spinal-cord neurodegenerative disorder of late onset, characterized by progressive weakness and spasticity of the lower limbs, and, more recently, a homozygous missense mutation, D29G, in HSPD1, encoding the mitochondrial Hsp60 chaperonin, causing a mitochondrial Hsp60 chaperonopathy linked to brain hypomyelination and leukodystrophy. This finding provides evidence for the pivotal role of the mitochondrial Hsp60 chaperonin in the process of normal brain myelination and in the pathogenesis of hypomyelinating neurodegenerative disease [58]. Notably, we found increased expression of thioredoxin in MS as compared to control subjects. To date this is the first report demonstrating changes in thioredoxin/thioredoxin reductase protein levels in MS patients. Thioredoxin (TRX) represents an intracellular redox regulator that has been shown to be important for the regulation of redox-sensitive transcription factors [18]. In its reduced state TRX can oxidatively reactivate inactive transcription factors such as Jun, Fos, AP-1, redox factor-1 (ref-1), and Nrf-2 [59]. Thioredoxin reductase (TRXr) is a flavoprotein that catalyzes the NADPH-dependent reduction of oxidized thioredoxin protein. It is usually located in the cytosol, but it translocates into the nucleus in response to various stimuli associated with oxidative stress. TRXr plays a pivotal role in protecting against oxidative stress and in regulating cell growth and cell death. Constitutive TRXr expression has been observed in several cell types of the mammalians, including neuronal cells after nitrosative stress. Both in vivo and in vitro studies demonstrated that TRX and TRXr have protective roles against cytotoxicity mediated by the generation of ROS [59]. Notably, in our study we demonstrate that thioredoxin reductase is decreased in MS lymphocytes. This may be relevant to the pathogenesis of MS, as we have previously demonstrated in CSF and plasma of MS patients a significant decrease of GSH/GSSG ratio $[5,9]$. Consistent with this notion, it is well established that cellular redox regulation of many processes is provided by the cooperation between the Trx and glutathione systems [60]. In fact, Trx and GSH systems are involved in a variety of redox-dependent reactions, such as supplying reducing equivalents for ribonucleotide reductase, and peptide methionine sulfoxide reductase, the latter being involved in antioxidant defense and regulation of the cellular redox state [59]. Therefore, Trx and GSH form a powerful system controlling redox regulation of gene expression, signal transduction, cell proliferation, protection against oxidative stress, antiapoptotic functions, growth factor and co-cytokine effects, as well as regulation of the redox state of the extracellular environment $[61,62]$. In addition, increased Trx- 1 expression has been described in many human primary cancers and tumor cell lines [63]. Changes in MS redox status observed in this study were reflected also by the interesting finding that Sirtuin-1 is present in the plasma of MS patients at significantly higher levels compared to control subjects. As far as we are concerned this is the first evidence of increased Sirtuin-1 expression in the blood of MS patients. Sirtuins are a family of histone deacetylase, that, in humans, includes at least seven members, with different cellular and subcellular localization and substrate binding activity $[15,61][15,64]$. The most studied is the SIRT1, a NAD+ dependent enzyme, involved in deacetylation of different proteins and in the regulation of energy metabolism and redox state. Sirtuins are involved in the regulation of important biological processes, such as apoptosis, cell differentiation, energy transduction, glucose homeostasis $[18,65]$, as well as in mediating enhancement of synaptic plasticity and neurogenesis in response to exercise, dietary energy restriction, and other hormetic environmental factors $[15,66]$. Because of their roles in cellular stress responses, sirtuins would be expected to play particularly important roles in adaptive responses of neural cells to stress, such as that associated with the MS pathogenesis. In support of this conceivable possibility, SIRT1 has been shown to interact either directly or indirectly with several pathways known to be involved in adaptive neural plasticity, including the $\mathrm{Ca}^{2+}$-calmodulin-cyclic adenosine monophosphate (AMP) response element binding protein and NF-kB systems [15]. Thus, our finding may be relevant to the MS pathogenesis, in view of the fact that SIRT1 are emerging as a promising candidate target for therapeutic interventions in metabolic and neurodegenerative disorders [67].

MS is a progressive inflammatory neurodegenerative disease exhibiting a variable clinical presentation which has no 'diagnostic' laboratory test; this often results in delays to definite diagnosis. In confronting the disease, early diagnosis and appropriate, timely therapeutic intervention are critical factors in ensuring favorable long-term outcomes. The availability of reliable biomarkers could radically alter our management of MS at critical phases of the disease spectrum. Identification of markers that could predict the development of MS in high-risk populations would allow for intervention strategies that may prevent evolution to definite disease. Ongoing work with biomarkers that reflect drug bioavailability and factors that distinguish between medication responders and nonresponders are also under investigation. Pharmacological modulation of cellular stress pathways has emerging implications in the treatment of several human diseases [15]. Consistent with this notion, inactivating polymorphisms of genes encoding detoxification enzymes, such as $\mathrm{NAD}(\mathrm{P}) \mathrm{H}$ :quinone oxidoreductase 1 (NQO1), has been recently reported to be associated with increased susceptibility to MS, suggesting a possible role for the NQO1 genetic background in the development of primary progressive MS [68]. Based on the overlapping inducibility of the cytoprotective phase 2 response and the vitagene network, there is now proven explanation for sensing mechanism of the initial chemical signal that ultimately leads to enhanced gene expression. Thus, modulation of endogenous cellular defense mechanisms via the stress response signaling represents an innovative approach to therapeutic intervention in diseases causing tissue damage, such as neurodegeneration. This is accomplished by a complex network of the so-called longevity assurance processes, which are composed of several genes termed vitagenes [15]. Maintaining or recovering the activity of vitagenes through nutritional interventions or specifically active drugs will help uncover novel clues for neuroprotective therapeutic strategies with relevance for clinical applications well beyond the field of MS alone.

\section{Acknowledgments}

Work from the authors' laboratories was supported by grants from MIUR, FIRB RBRN07BMCT, I.N.B.B., and by "Fondi Ateneo" 2008 and 2009. 
CV would like to thank Giorgio Federici for helpful discussions during the genesis of this article.

\section{References}

[1] Compston A, Coles A. Multiple sclerosis. Lancet 2008;372:1502-17.

[2] Nessler S, Brück W. Advances in multiple sclerosis research in 2009. J Neurol 2010;257:1590-3.

[3] Derwenskus J. Current disease-modifying treatment of multiple sclerosis. Mt Sinai J Med 2011;78:161-75.

[4] Tumani H, Hartung HP, Hemmer B, Teunissen C, Deisenhammer F, Giovannoni. et al. Cerebrospinal fluid biomarkers in multiple sclerosis. Neurobiol Dis 2009;35:117-27.

[5] Calabrese V, Scapagnini G, Ravagna A, Bella R, Foresti R, Bates TE, et al. Nitric oxide synthase is present in the cerebrospinal fluid of patients with active multiple sclerosis and is associated with increases in cerebrospinal fluid protein nitrotyrosine and S-nitrosothiols and with changes in glutathione levels. J Neurosci Res 2002;70:580-7.

[6] Witherick J, Wilkins A, Scolding N, Kemp K. Mechanisms of oxidative damage in multiple sclerosis and a cell therapy approach to treatment. Autoimmune Dis 2010;2011:164608.

[7] Ziemann U, Wahl M, Hattingen E, Tumani H. Development of biomarkers for multiple sclerosis as a neurodegenerative disorder. Prog Neurobiol 2011 [Epub April 16].

[8] Van Noort JM, van den Elsen PJ, van Horssen J, Geurts JJ, van der Valk P, Amor S. Preactive multiple sclerosis lesions offer novel clues for neuroprotective therapeutic strategies. CNS Neurol Disord Drug Targets 2011;10:68-81.

[9] Calabrese V, Scapagnini G, Ravagna A, Bella R, Butterfield DA, Calvani M, et al. Disruption of thiol homeostasis and nitrosative stress in the cerebrospinal fluid of patients with active multiple sclerosis: evidence for a protective role of acetylcarnitine. Neurochem Res 2003;28:1321-8.

[10] Mattsson N, Yaong M, Rosengren L, Blennow K, Månsson JE, Andersen O, et al. Elevated cerebrospinal fluid levels of prostaglandin E2 and 15-(S)-hydroxyeicosatetraenoic acid in multiple sclerosis. J Intern Med 2009;265:459-64.

[11] Mattsson N, Haghighi S, Andersen O, Yao Y, Rosengren L, Blennow K, et al. Elevated cerebrospinal fluid F2-isoprostane levels indicating oxidative stress in healthy siblings of multiple sclerosis patients. Neurosci Lett 2007;414: 233-6.

[12] Calabrese V, Bella R, Testa D, Spadaro F, Scrofani A, Rizza V, et al. Increased cerebrospinal fluid and plasma levels of ultraweak chemiluminescence are associated with changes in the thiol pool and lipid-soluble fluorescence in multiple sclerosis: the pathogenic role of oxidative stress. Drugs Exp Clin Res 1998;24:125-31.

[13] Calabrese V, Cornelius C, Dinkova-Kostova AT, Calabrese EJ. Vitagenes, cellular stress response and acetylcarnitine: relevance to hormesis. Biofactors 2009;35:146-60.

[14] Calabrese V, Cornelius C, Rizzarelli E, Owen JB, Dinkova-Kostova AT, Butterfield DA. Nitric oxide in cell survival: a Janus molecule. Antioxid Redox Signal 2009;11:2717-39.

[15] Calabrese V, Cornelius C, Dinkova-Kostova AT, Calabrese EJ, Mattson MP. Cellular stress responses, the hormesis paradigm, and vitagenes: novel targets for therapeutic intervention in neurodegenerative disorders. Antioxid Redox Signal 2010;13:1763-811.

[16] Calabrese V, Cornelius C, Stella AM, Calabrese EJ. Cellular stress responses, mitostress and carnitine insufficiencies as critical determinants in aging and neurodegenerative disorders: role of hormesis and vitagenes. Neurochem Res 2010;35:1880-915.

[17] Calabrese V, Mancuso C, Calvani M, Rizzarelli E, Butterfield DA, Giuffrida Stella AM. Nitric oxide in the CNS: neuroprotection versus neurotoxicity. Nat Neurosci 2007;8:766-75.

[18] Calabrese V, Cornelius C, Mancuso C, Pennisi G, Calafato S, Bellia F, et al. Cellular stress response: a novel target for chemoprevention and nutritional neuroprotection in aging, neurodegenerative disorders and longevity. Neurochem Res 2008;33:2444-71.

[19] McDonald WI, Compston A, Edan G, Goodkin D, Hartung HP, Lublin FD, et al. Recommended diagnostic criteria for multiple sclerosis: guidelines from the International Panel on the diagnosis of multiple sclerosis. Ann Neurol 2001;50(1):121-7.

[20] Lublin FD, Reingold SC. Defining the clinical course of multiple sclerosis: results of an international survey National Multiple Sclerosis Society (USA) Advisor Committee on Clinical Trials of New Agents in Multiple Sclerosis. Neurology 1996;46:907-11.

[21] Smith P, Krohn R, Hermanson G, Mallia A, Gartner F, Provenzano M, et al. Measurement of proteins using bicinchoninic acid. Ann Biochem 1985;150: 76-85.

[22] Calabrese V, Guagliano E, Sapienza M, Panebianco M, Calafato S, Puleo E, et al. Redox regulation of cellular stress response in aging and neurodegenerative disorders: role of vitagenes. Neurochem Res 2007;32:757-73.

[23] Calabrese V, Boyd-Kimball D, Scapagnini G, Butterfield DA. Nitric oxide and cellular stress response in brain aging and neurodegenerative disorders: the role of vitagenes. In Vivo 2004;18:245-67.

[24] Mancuso C, Scapagnini G, Curro D, Giuffrida Stella AM, De Marco C, Butterfield response in neurodegenerative disorders. Front Biosci 2007;12:1107-23.
[25] Butterfield DA, Stadtman ER. Protein oxidation processes in aging brain. Adv Cell Aging Gerontol 1997;2:161-91.

[26] Lauderback CM, Hackett JM, Huang FF, Keller JN, Szweda LI, Markesbery WR et al. The glial glutamate transporter, GLT-1, is oxidatively modified by 4 hydroxy-2-nonenal in the Alzheimer's disease brain: role of A-beta 1-42. Neurochem 2001;78:413-6.

[27] Butterfield DA, Howard BJ, Yatin S, Allen KL, Carney JM. Free radical oxidation of brain proteins in accelerated senescence and its modulation by N-tertbutyl-phenylnitrone. Proc Natl Acad Sci U S A 1997;94:674-8.

[28] Tudor C, Lerner-Marmarosh N, Engelborghs Y, Gibbs PE, Maines MD. Biliverdin reductase is a transporter of haem into the nucleus and is essential for regulation of HO-1 gene expression by haematin. Biochem J 2008;413:405-16.

[29] Yoneyama-Sarnecky T, Olivas AD, Azari S, Ferriero DM, Manvelyan HM, NobleHaeusslein LJ. Heme oxygenase-2 modulates early pathogenesis after traumatic injury to the immature brain. Dev Neurosci 2010;32:81-90.

[30] Mao P, Reddy PH. Is multiple sclerosis a mitochondrial disease? Biochim Biophys Acta 2010;1802:66-79.

[31] Frohman EM, Racke MK, Raine CS. Multiple sclerosis-the plaque and its pathogenesis. N Engl J Med 2006;354:942-55.

[32] Pokryszko-Dragan A, Gruszka E, Bilinśka M. Secondary progressive multiple sclerosis-clinical course and potential predictive factors. Neurol Neurochir Pol 2008;42:6-11.

[33] Gonsette RE. Neurodegeneration in multiple sclerosis: the role of oxidative stress and excitotoxicity. J Neurol Sci 2008;274:48-53.

[34] Turturici G, Sconzo G, Geraci F. Hsp70 and its molecular role in nervous system diseases. Biochem Res Int 2011;2011:618127.

[35] Gonsette RE. Oxidative stress and excitotoxicity: a therapeutic issue in multiple sclerosis? Mult Scler 2008;14:22-34.

[36] Lindsey JW, Agarwal SK, Tan FK. Gene expression changes in multiple sclerosis relapse suggest activation of T and non-T cells. Mol Med 2011;17:95-102.

[37] Gonsette RE. Endogenous neuroprotection in multiple sclerosis. Acta Neurol Belg 2010;110:26-35.

[38] Calabrese V, Raffaele R, Cosentino E, Rizza V. Changes in cerebrospinal fluid levels of malondialdehyde and glutathione reductase activity in multiple sclerosis. Int J Clin Pharmacol Res 1994;14:119-23.

[39] Miller E, Mrowicka M, Saluk-Juszczak J, Ireneusz M. The level of isoprostanes as a non-invasive marker for in vivo lipid peroxidation in secondary progressive multiple sclerosis. Neurochem Res 2011;36(6):1012-6.

[40] Choi IY, Lee SP, Denney DR, Lynch SG. Lower levels of glutathione in the brains of secondary progressive multiple sclerosis patients measured by $1 \mathrm{H}$ magnetic resonance chemical shift imaging at 3 T. Mult Scler 2011;17:289-96.

[41] Liu Y, Liu J, Tetzlaff W, Paty DW, Cynader MS. Biliverdin reductase, a major physiologic cytoprotectant, suppresses experimental autoimmune encephalomyelitis. Free Radic Biol Med 2006;40:960-7.

[42] Leung G, Sun W, Zheng L, Brookes S, Tully M, Shi R. Anti-acrolein treatment improves behavioral outcome and alleviates myelin damage in experimental autoimmune encephalomyelitis mouse. Neuroscience 2011;173:150-5.

[43] Esposito E, Cuzzocrea S. Antiinflammatory activity of melatonin in central nervous system. Curr Neuropharmacol 2010;8(September (3)):228-42.

[44] Hultqvist M, Olofsson P, Holmberg J, Backstrom BT, Tordsson J, Holmdahl R. Enhanced autoimmunity, arthritis, and encephalomyelitis in mice with a reduced oxidative burst due to a mutation in the Ncf1 gene. Proc Natl Acad Sci USA 2004;101:12646-51.

[45] Dello Russo C, Polak PE, Mercado PR, Spagnolo A, Sharp A, Murphy P, et al. The heat-shock protein 90 inhibitor 17-allylamino-17-demethoxygeldanamycin suppresses glial inflammatory responses and ameliorates experimental autoimmune encephalomyelitis. J Neurochem 2006;99:1351-62.

[46] Saha RN, Pahan K. Regulation of inducible nitric oxide synthase gene in glial cells. Antioxid Redox Signal 2006;8:929-47.

[47] Bolaños JP, Heales SJ. Persistent mitochondrial damage by nitric oxide and its derivatives: neuropathological implications. Front Neuroenergetics 2010;2 (February):1.

[48] Gibbs PE, Maines MD. Biliverdin inhibits activation of NF-kappaB: reversal of inhibition by human biliverdin reductase. Int J Cancer 2007;121:2567-74.

[49] Maines MD. The heme oxygenase system: past, present, and future. Antioxid Redox Signal 2004;6:797-801.

[50] Calabrese V, Butterfield DA, Scapagnini G, Stella AM, Maines MD. Redox regulation of heat shock protein expression by signaling involving nitric oxide and carbon monoxide: relevance to brain aging, neurodegenerative disorders, and longevity. Antioxid Redox Signal 2006;8:444-77.

[51] Motterlini R, Foresti R, Bassi R, Calabrese V, Clark JE, Green CJ. Endothelial heme oxygenase- 1 induction by hypoxia. Modulation by inducible nitricoxide synthase and S-nitrosothiols. J Biol Chem 2000;275:13613-20.

[52] Calabrese V, Butterfield DA, Stella AM. Aging and oxidative stress response in the CNS. In: Abel L, Regino P-P, Steffen R, editors. Development and Aging Changes in the Nervous System. Handbook of Neurochemistry and Molecular Neurobiology. 2008. p. 128-234.

[53] Calabrese V, Scapagnini G, Colombrita C, Ravagna A, Pennisi G, Giuffrida Stella $\mathrm{AM}$, et al. Redox regulation of heat shock protein expression in aging and neurodegenerative disorders associated with oxidative stress: a nutritional approach. Amino Acids 2003;25:437-44.

[54] Cid C, Alcazar A. Protection of oligodendrocyte precursor cells by low doses of HSP90 inhibitors in cell culture. Exp Neurol 2010;225:29-33.

[55] Mháille AN, McQuaid S, Windebank A, Cunnea P, McMahon J, Samali A, et al. Increased expression of endoplasmic reticulum stress-related signaling pathway molecules in multiple sclerosis lesions. J Neuropathol Exp Neurol 2008;67:200-11. 
[56] Stahnke T, Stadelmann C, Netzler A, Brück W, Richter-Landsberg C. Differential upregulation of heme oxygenase-1 (HSP32) in glial cells after oxidative stress and in demyelinating disorders. J Mol Neurosci 2007;32: 25-37.

[57] Bender T, Lewrenz I, Franken S, Baitzel C, Voos W. Mitochondrial enzymes are protected from stress-induced aggregation by mitochondrial chaperones and the Pim1/LON protease. Mol Biol Cell 2011;22(March (5)):541-54.

[58] Magen D, Georgopoulos C, Bross P, Ang D, Segev Y, Goldsher D, et al. Mitochondrial hsp60 chaperonopathy causes an autosomal-recessive neurodegenerative disorder linked to brain hypomyelination and leukodystrophy. Am J Hum Genet 2008;83:30-42.

[59] Calabrese V, Cornelius C, Mancuso C, Barone E, Calafato S, Bates T, et al. Vitagenes, dietary antioxidants and neuroprotection in neurodegenerative diseases. Front Biosci 2009;14:376-97.

[60] Mandal PK, Seiler A, Perisic T, Kölle P, Banjac Canak A, Förster H, et al. System $\mathrm{x}(\mathrm{c})$ - and thioredoxin reductase 1 cooperatively rescue glutathione deficiency. J Biol Chem 2010;285:22244-53.

[61] Lowes DA, Galley HF. Mitochondrial protection by the thioredoxin-2 and glutathione systems in an in vitro endothelial model of sepsis. Biochem 2011;436:123-32.
[62] Bell KF, Hardingham GE. CNS peroxiredoxins and their regulation in health and disease. Antioxid Redox Signal 2011;14:1467-77.

[63] Qu Y, Wang J, Ray PS, Guo H, Huang J, Shin-Sim M, et al. Thioredoxin-like 2 regulates human cancer cell growth and metastasis via redox homeostasis and NF-кB signaling. J Clin Invest 2011;121:212-25.

[64] Dali-Youcef N, Lagouge M, Froelich S, Koehl C, Schoonjans K, Auwerx J. Sirtuins: the 'magnificent seven', function, metabolism and longevity. Ann Med 2007;39:335-45.

[65] Yi J, Luo J. SIRT1 and p53, effect on cancer, senescence and beyond. Biochim Biophys Acta 2010;1804:1684-9.

[66] Min SW, Cho SH, Zhou Y, Schroeder S, Haroutunian V, Seeley WW, et al. Acetylation of tau inhibits its degradation and contributes to tauopathy. Neuron 2010;67:953-66.

[67] Herranz D, Serrano M. SIRT1: recent lessons from mouse models. Nat Rev Cancer 2010;10:819-23.

[68] Stavropoulou C, Zachaki S, Alexoudi A, Chatzi I, Georgakakos VN, Terzoudi GI, et al. The $C(609) \mathrm{T}$ inborn polymorphism in $\mathrm{NAD}(\mathrm{P}) \mathrm{H}$ :quinone oxidoreductase 1 is associated with susceptibility to multiple sclerosis and affects the risk of development of the primary progressive form of the disease. Free Radic Biol Med 2011;51:713-8. 